\title{
An annotated checklist of Chironomidae (Diptera) trapped in Brittany (France) since 1975
}

\author{
Y. R. Delettre ${ }^{1}$
}

Keywords : Diptera, Chironomidae, species diśtribution, France.

The chironomid fauna ot the Western part of France has been notably understudied ; few data are available on species distribution for the region. This paper provides some information obtained from several studies conducted in the eastern part of Brittany. Although a small part of this region is considered, the checklist comprises 94 species, four of which are recorded for the first time in France.

\section{Liste annotée des Chironomidae (Diptera) capturés en Bretagne (France) depuis 1975}

Mots-clés : Diptera, Chironomidae, répartition, France.

Les Chironomidae de l'Ouest de la France ont été particulièrement peu étudiés par le passé et la distribution des espèces est donc fort méconnue. Cette publication recense les informations collectées par une série d'études menées dans la partie Est de la Bretagne. Bien que seule une partie de cette région ait été prise en compte; la liste comporte 94 espèces dont quatre sont nouvelles pour la France.

\section{Introduction}

The biogeographical distribution of Chironomidae (Diptera) in France and Corsica was synthesized by Serra-Tosio \& Laville (1991) and some additions and corrections were published by Laville \& Serra-Tosio (1996). As a result, 646 species were listed from France, accounting for one half of the total chironomid fauna in the Palaearctic. A recent paper by Garcia \& Laville (2001) added 12 new species to this list.

However, as underlined by these authors, some regions were more sampled than others and the study of lowland plains, which belong to the $13^{\text {th }}$ biogeographical region (Illies 1978), is rather fragmentary. The subregion A13, as defined by Serra-Tosio \& Laville (1991), includes Brittany, Normandy and the northern part of France (Fig. 1). In Brittany, very few limnological studies have been conducted, and thus, the chironomid fauna is still poorly known.

1 . C.N.R.S., U.M.R 6553 "Ecobio", University of Rennes I. Biological Field Research Station, F-35380 Paimpont (France). E-mail: yannick.delettre@univ-rennes1.fr
The current paper provides a list of species derived from different research works conducted in the eastern part of Brittany since 1975. Most studies were focused on terrestrial Chironomidae, although some of them also considered aquatic habitats. However, the study of flying insects throughout three agricultural landscapes provided catches of species inhabiting various water bodies, which enlarged the field of investigation of the current work. No separate list of species has been previously published for Brittany.

\section{Methods}

Two main sampling methods were used, namely emergence traps (E) and yellow water traps (Y). The former catch adult insects emerging from water bodies or from the soil, and thus allow an accurate definition of specific larval habitats. The latter catch flying adults searching for propitious swarming, resting or ovipositing sites, yielding a mixture of species originating in several habitats; the resulting list contains a spectrum of species living in a given heterogeneous landscape. Additionally, a skim net (Sk) was used to collect pupal exuviae in some pools. Samples were preserved in ethanol $\left(70^{\circ}\right)$, and then determined under a stereomicroscope using appropriate keys and revisions (see $\mathrm{Re}-$ ferences). 


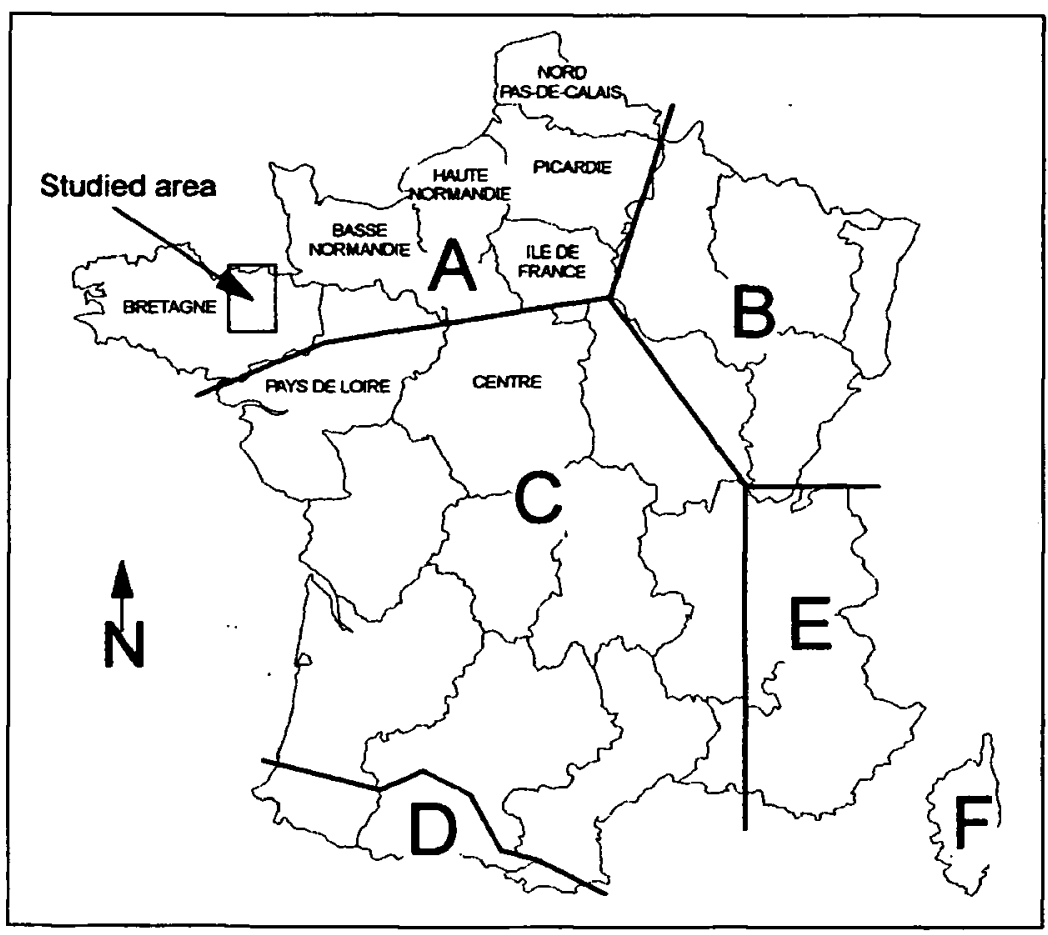

Fig.1. A schematic map of biogeographical sub-regions of France, adapted from Serra-Tosio \& Laville (1991). The study area considered in Brittany is shown.

Fig. 1. Carte schématique des sous-régions biogéographiques de France, adaptée de Serra-Tosio \& Laville (1991) montrant la zone d'étude en Bretagne.

\section{Study sites}

A total of 12 different sites was studied, most of them for more than one year. All are located in the eastern part of Brittany (Ille \& Vilaine and Morbihan), at low altitude (below $250 \mathrm{~m}$ a.s.l.) and at less than $80 \mathrm{~km}$ from coasts. They can be clustered in five sets, as follows:

1. Three different heathland types (dry, mesic and tall heathlands) located at Trécesson (Morbihan), near the Forest of Paimpont, either undisturbed or subjected to prescribed fire, sampled with emergence traps for four years (columns [1] to [4] in Table 1). Results for burnt patches are pooled (column [4]). All these heathland patches are located on purple schistose rocks. Soils are acidic brown soils. The mesic heathland is established on a hydromorphic soil. The vegetation is typical of heathlands, including furze, heather and various gramineous plants as well as lichens. The speed of vegetation re-establishment changed according to fire intensity. Detailed results can be found in Delettre $(1994,1995)$.
2. Five different habitats bordering a pond in the same forest, namely the pond banks, a riparian woodland, a small meadow and, farther, acidic swards and forest plots (columns [5] to [9] in Table 1). These habitats were sampled weekly for two years (Delettre, et al. 1992, Delettre 1993). Acidic swards (dry heathlands) located on schistose rocks were more extensively studied during six years in the same area (Delettre 1994). All of them were flooded in winter owing to abundant rainfall and low soil permeability. Solar radiation and reduced soil thickness induced a deep soil drought every summer on those sites. Both emergence traps and yellow water traps were used.

3. Two acidic ponds (Etang du Châtenay \& Etang de Comper) in the same area near the Paimpont biological field research Station, where a skim net was occasionally used in spring (Delettre unpubl.). Their physical and chemical characteristics were not determined. Species occurring at these sites are recorded in column [10] (Table 1).

4. A temporary pond, located in an old abandoned quarry, studied for 15 months by Ducrotoy (1976) and for two years by Delettre $(1984,1989)$. According to Ducrotoy (1976), its main characteristics were : area $=300 \mathrm{~m}^{2}$ in winter, maximum depth $=24 \mathrm{~cm}$, average $\mathrm{pH}=5.1$, conductivity $=$ $0.02 \mu \Omega$, calcium $=0.62 \mathrm{mg} / \mathrm{l}$, phosphate $=0$, nitrate $=0.017 \mathrm{mg} / \mathrm{l}$, oxygen saturation $=74-100 \%$ all through the year, average annual temperature $=10.9^{\circ} \mathrm{C}$, organic matter of the bottom sediment $=1-4 \%, \mathrm{C} / \mathrm{N}=11.5$. Mud granulometry was dominated by silt (32-43\%) and sand (31-49\%) while clay accounted for $18-23 \%$ (Delettre 1984). The pond dried up each summer for one week to two months depending on the year (Delettre 1989). Both emergence traps and yellow water traps were used (column [11] in Table 1).

5. Three agricultural landscapes with dense to sparse hedgerow networks, located south of Mont Saint Michel Bay (Ille \& Vilaine, $48^{\circ} 36^{\prime} \mathrm{N}, 1^{\circ} 32 \mathrm{~W}$ ) in which a total of 128 yellow water traps was used from March to July for two consecutive years. These landscapes (each covering 500-700 ha) include permanent streams running across an agricultural mosaic (corn or wheat fields, permanent and temporary meadows, fallow land, woodlots). A synthesis of some results can be found in Burel et al. $(1998,2000)$ and a detailed analysis of aquatic species is provided in Delettre \& Morvan (2000). See column [12] in Table 1. 


\section{Results}

As a whole, 94 species were identified (Table 1). The Orthocladiinae accounted for the greatest part (58 spp.), followed by the Chironominae ( $24 \mathrm{spp}$.) and Tanypodinae (11 spp.) ; only one species belonging to the sub-family Prodiamesinae was recorded. Four species were new for France, 50 were new for sub-region A13, three species identifications were confirmed and one species, omitted in Laville \& Serra-Tosio (1996), was added.

Species new for France are four terrestrial Orthocladiinae : Pseudosmittia angusta (Edw.), P. curticosta (Edw.), Smittia contingens (Walk.) and S. foliacea (K.). All of them have been recorded previously from England (Pinder 1978). S. foliacea is also known from Belgium (Goddeeris \& Behen 1991). P. curticosta (Edw.) accounted (as Smittia curticosta Edw.) for ca. $50 \%$ of the total fly fauna in a brown woodland soil (mull humus type) in England (Healey \& Russel-Smith 1971). Species new for A13 sub-region are indicated in Table 1. Most of them were already found in other French sub-regions (e.g. B13 or C13). One species (Smittia celtica Rossaro \& Delettre) was, till now, only found on acidic swards in Brittany (Rossaro \& Delettre 1992). It was tentatively reported as Parasmittia sp. nr. carinata Str. by Delettre in some previous papers. Its ecology, population dynamics and ecophysiological capabilities were extensively studied by Delettre (1984, 1988a, 1988b).

Several remarks must be added. Bryophaenocladius brincki (Freem.) was described from South Africa by Freeman (1955) and redescribed by Saether (1973) from Mt. Kenya (8-9000 ft. a.s.l.). Identification of our specimens was checked by Saether, who concluded the identification accurate (O.A. Saether, pers. comm). The occurrence of this species so far from its supposed biogeographic area was surprising, but most terrestrial chironomid species are highly understudied and many terrestrial ecosystems have not been sampled for this taxon. Therefore, numerous gaps in the biogeographical distribution of edaphic species are likely to occur frequently. This remark is also valid for the four terrestrial species mentioned above.

Although Saether (1983) suggested that Raphidocladius (a subgenus of Gymnometriocnemus) was probably aquatic or semi-aquatic, $G$. (R.) brumalis (Edw.) was found in huge numbers in fully terrestrial habitats including dry heathlands and woodlands as well.

Limnophyes minimus $(\mathrm{Mg}$.) and L. natalensis $(\mathrm{K}$.) were found together at several sites in Brittany. According to Ole Saether (pers. comm.), who examined our specimens, hybrids are likely to occur between these two species in Brittany. The same problem was mentioned recently by Henk Moller-Pillot (pers. comm.) in the Netherlands.

Amongst the aquatic species, most are tyical of ponds and small rivers. Species originating in springs are not well represented, except Rheocricotopus ( $s$. str.) effusus and Chaetocladius dentiforceps (Edw.), which is typical of limnocrene springs. Numerous adults of the latter species emerged from the small temporary pond located in an old quarry. Heterotrissocladius marcidus (Walk.) is an hyporheic species. Other species living in interstitial water are likely to occur but their study needs appropriate sampling methods (Marmonier et al., 2000). Cladotanytarsus nigrovittatus (G.), already known from U.K. and Belgium, is not listed in Serra-Tosio \& Laville (1991). It was found in lakes above $1000 \mathrm{~m}$ a.s.l. in the Pyrenees by Moubayed et al. (1999).

Some other species are not listed in the current paper, as several specimens were too deteriorated or found at the pupal stage, which did not allow an accurate identification. They belong to the following senera : Conchapelopia, Krenosmittia and Tanypus.

\section{Conclusion}

The current list of Chironomidae from Brittany is, of course, far from complete as many aquatic habitats have not yet been investigated. Many species recorded in other lowland areas in France are likely also to be found in Brittany, when detailed limnological studies of running and standing waters in the rest of Brittany are performed. This work is planned for the near future, including the interstitial fauna, and thus additions to the current list will be provided later. However, this checklist does show the value of studying the most western part of France and the need for future research.

\section{Acknowledgements}

I am indebted to Ole A. Saether and B. Serra-Tosio for their help in identifying several species. A special thank to Ronald Wilson who helped me starting the study of pupal exuviae during his stay in our laboratory in 1992. I also want to thank Peter Langton and Joel Moubayed for their helpful comments on the manuscript. 
Table 1. Checklist of chironomid species. Status (St): \# = occurrence confirmed in A13; ${ }^{*}=$ new species in A13 but previously found in other subregions of France; $F=$ new for France; $O=$ omitted in Laville \& Serra-Tosio (1996). Trapping devices as follows : $\mathrm{E}=$ emergence trap; $\mathrm{Y}=$ Yellow pan trap; $\mathrm{Sk}=$ skim net.

Tableau 1. Liste annotée des espèces de Chironomidés. Situation $(\mathrm{St})$ : \# présence confirmée en $\mathrm{A} 13 ;{ }^{*}=$ espèce nouvelle en $\mathrm{A} 13$ mais connue antérieurement d'autres sous-régions de France ; $\mathrm{F}=$ nouvelle pour la France ; $\mathrm{O}=$ oubliée chez Laville \& SerraTosio (1996). Appareils de récoltes : $\mathrm{E}$ : piège à émergence ; $\mathrm{Y}=$ piège jaune ; $\mathrm{Sk}=$ filet à écume.

SPECIES

\begin{tabular}{|c|c|c|c|c|c|c|c|c|c|c|c|c|}
\hline t. & 1 & 2 & 3 & 4 & 5 & 6 & 7 & 8 & 9 & 10 & 11 & 12 \\
\hline 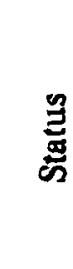 & 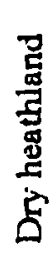 & 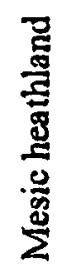 & 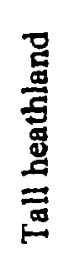 & 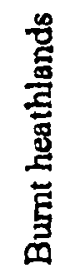 & 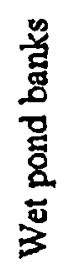 & 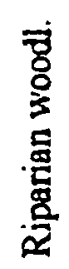 & 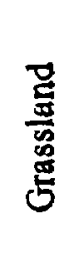 & 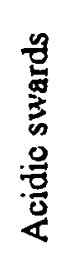 & 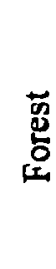 & 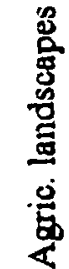 & : & 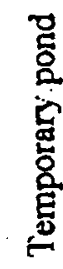 \\
\hline
\end{tabular}

\section{Tanypodinae}

Ablabesmyia longistyla Fitt.

$\mathbf{Y}$

Ablabesmyia monilis (L.)

$\mathbf{Y}$

Y

E

Arciopelopia barbitarsis (Zet1.)

Macropelopia adaucta (K.)

Macropelopia nebulosa (Mg.)

Y

Sk

Macropelopia notata (Mg.)

$\mathbf{Y}$

Monopelopia temuicalcar (K)

$Y$

E

Paramerina cingulata (Walk)

Y

$\mathbf{Y}$

Procladius (Holotanypus) choreus (Mg.)

Sk E

Procladius (Holotanypus) crassinervis (Zett)

Psectrotanypus varius (Fabr.)

\section{Prodiamesinae}

Prodianesa olivacea (Mg.)

Orthocladiinae

Acricotopus kucens (Zett.)

Brillia modesta (Mg.)

Bryophnenocladius brincki (Freem.)

Bryophaenocladius muscicala (K.)

Bryophaenoclodius subvernalis (Edw.)

Bryophaenocladius vermalis (G.)

Camplocladius stercorarius (de Geer)

Chaetocladius dentiforceps (Edw.)

Chaetocladius perennis (Mg.)

Chaetocladius piger (G.)

Conyoneura cellica Edw.

Corvnoneura ectwardsi Brundin

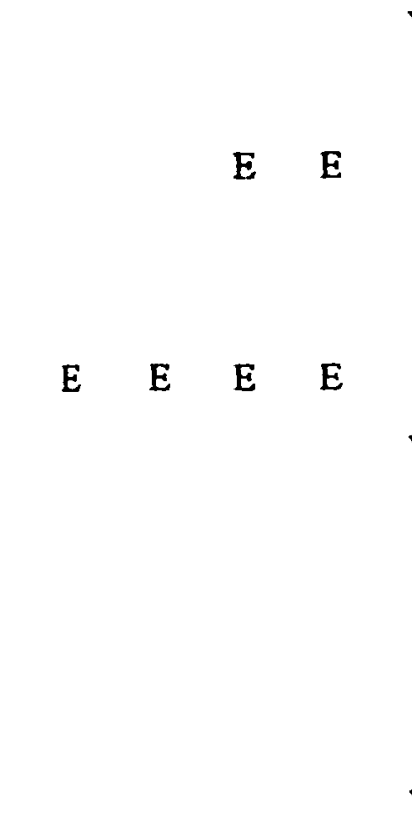

Y

$\mathbf{Y}$

$\mathbf{Y}$

$Y$

E

$Y$

$\begin{array}{llll}Y & E & Y & Y\end{array}$

$\mathbf{Y} \quad \mathbf{Y}$

Y Y

E

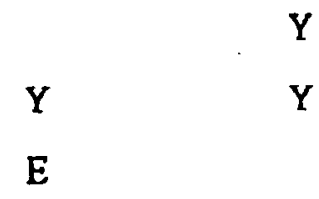

$Y$ 
Table 1. (continued).

Tableau 1. (suite).

\begin{tabular}{|c|c|c|c|c|c|c|c|c|c|c|c|c|c|}
\hline & $\mathrm{St}$ & 1 & 2 & 3 & 4 & 5 & 6 & 7 & 8 & 9 & 10 & 11 & 12 \\
\hline Corynoneura laczustris Edw. & $\#$ & & & & & $\mathrm{Y}$ & & & & & & & \\
\hline Connnoneura lohata Edw. & * & & & & & & & $\mathrm{Y}$ & & & $\mathbf{Y}$ & & \\
\hline Cricotopus (s. str.) pulchripes Verr. & $*$ & & & & & & & $\mathbf{Y}$ & & & & & \\
\hline Cricotopus (Isocladius) sylvestris (Fabr.) & & & & & & & & $\mathbf{Y}$ & $\mathbf{Y}$ & & & & \\
\hline Diplocladius cultiger $\mathrm{K}$ & $*$ & & & & & & & $\mathbf{Y}$ & & & & & \\
\hline Epoicocladius flavens (Malloch) & $\bullet$ & & & & & $\mathrm{Y}$ & & & & & $\mathrm{Y}$ & & \\
\hline Enkiefferiella brevicalcar $(\mathrm{K})$ & * & & & & & & & $\mathbf{Y}$ & & & $\mathbf{Y}$ & & \\
\hline $\begin{array}{l}\text { Gymnometriocnemus (Raphidocladius) brumalis } \\
\text { (Edw.) }\end{array}$ & & $E$ & $\mathrm{E}$ & $\mathrm{E}$ & $E$ & & $\mathrm{E}$ & & & $\mathrm{E}$ & $\mathrm{Y}$ & & $\mathrm{E}$ \\
\hline Heterotanytarsus apicalis (K.) & * & & & & & Y & $\mathrm{Y}$ & $\mathbf{Y}$ & $\mathrm{Y}$ & $\mathrm{Y}$ & & Sk & \\
\hline Heterotrissocladius marcidus (Walk) & $*$ & & & & & $\mathbf{Y}$ & & & & & $\mathbf{Y}$ & & \\
\hline Limnophyes habilis (Walk) & $*$ & & & & & & $\mathrm{Y}$ & $Y$ & & $\mathrm{Y}$ & $\mathbf{Y}$ & $\cdot$ & \\
\hline Limnophyes minimus (Mg.) & & $\mathbf{E}$ & $E$ & $\mathbf{E}$ & $\mathbf{E}$ & EY & $\mathrm{E}$ & & $\mathrm{E}$ & $\mathbf{E}$ & $\mathbf{Y}$ & & $\mathbf{E}$ \\
\hline Limnophyes natalensis $(\mathrm{K}$ ) & & & $\mathrm{E}$ & & & $\mathrm{E}$ & & & & & & & \\
\hline Limnophyes pentaplasus $(\mathrm{K})$. & $*$ & & & & & & & & & & $Y$ & & \\
\hline Metriocnemus albolineatus (Mg.) & & & & & $E$ & $\mathrm{Y}$ & & & & & $\mathbf{Y}$ & & \\
\hline Metriocnemus fuscipes (Mg.) & 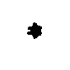 & & & & & & & $\mathbf{Y}$ & & & & & \\
\hline Metriocnemus obscuripes (Holm.) & $*$ & & & & & & $\mathbf{Y}$ & & & $\mathbf{Y}$ & $Y$ & & \\
\hline Metriocnemus tristellus Edw. & & & & & 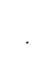 & & & $\mathbf{Y}$ & $\mathbf{Y}$ & $\mathbf{Y}$ & $\mathrm{Y}$ & & \\
\hline Nanocladius bicolor (Zett) & & & & & & & & & & & & Sk & \\
\hline Orthocladius (Eudactyloch) thienemanni $\mathrm{K}$ & * & & & & & & & & & & $\mathbf{Y}$ & & \\
\hline Paralimnophyes hudrophilus (G.) & & & & & & $\mathbf{Y}$ & & & & & $\mathrm{Y}$ & & E \\
\hline Parametriocnemus stylodus $(\mathrm{K}$ ) & & & & & & $\mathbf{Y}$ & & & $\mathbf{Y}$ & & $Y$ & & \\
\hline Paraphaenocladius impensus (Walk.) & * & & & & & $\mathbf{Y}$ & & & & & $\mathrm{Y}$ & & \\
\hline Paraphaenocladius irritus (Walk) & $*$ & & & & & & & & & & $\mathbf{Y}$ & & \\
\hline Paratrichocladius skinwithensis (Edw.) & * & & & & & & & & & & $Y$ & & \\
\hline Paratrissockadius excerptus (Walk.) & & & & & & $\mathbf{Y}$ & & & & & . & & \\
\hline Psectrocladius (Allopsectrocl.) obvius (Walk.) & $\bullet$ & & & & & & & & & & & Sk & \\
\hline Psectrocladius (s. str.) psiloplerus (K) & 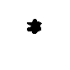 & & & & & $\mathbf{Y}$ & & & & & & Sk & \\
\hline Psectrocladius (s. str.) sordidellus (Zett) & & & & & & & & $\mathbf{Y}$ & & & & & \\
\hline $\begin{array}{l}\text { Psendorthocladius (s. str.) cranstoni Saeth. \& } \\
\text { Subl. }\end{array}$ & & & $E$ & & $\mathrm{E}$ & & & & & & & & \\
\hline Psetudorthocladius (s. str.) curtistylus (G.) & $\bullet$ & & & & & $\mathrm{E}$ & & & & & $\mathbf{Y}$ & & \\
\hline Pseudorthocladius (s. str.) filiformis (K.) & * & & & & & E & & & & & & & \\
\hline Pseudasmittia angusla (Edw.) & F & & & & & $\mathbf{E}$ & & & & & & & \\
\hline Pseudosmittia curticosta (Edw.) & F & & & & & EY & & & & & $Y$ & & \\
\hline Pseudasmituia longicrus $(\mathrm{K}$ ) & & & & & & & & & 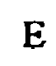 & & & & \\
\hline
\end{tabular}


Table 1. (continued).

Tableau 1. (suite).

\begin{tabular}{|c|c|c|c|c|c|c|c|c|c|c|c|c|c|}
\hline & St. & 1 & 2 & 3 & 4 & 5 & 6 & 7 & 8 & 9 & 10 & 11 & 12 \\
\hline Rheocricotopus (Psilocricot) atripes (K.) & * & & & & & & & & & & Y & & \\
\hline Rheocricotopus (Psilocricot.) glabricollis (Mg.) & - & & & & & & & & & & $\mathrm{Y}$ & & \\
\hline Rheocricotopus (s. str.) effiusus (Walk.) & * & & & & & & & & & & $\mathbf{Y}$ & & \\
\hline Rheocricotopus (s. str.) fuscipes (K) & & & & & & & & $\mathrm{Y}$ & & & Y & Sk & \\
\hline Smittia aterrima (Mg.) & & & & E & $\mathbf{E}$ & & & & & & Y & & \\
\hline Smittia celtica Rossaro \& Delettre & 0 & & & & & & & & $\mathrm{E}$ & & & & \\
\hline Smittia contingens (Walk) & $\mathrm{F}$ & & & & & & & $\mathrm{Y}$ & & Y & $\mathbf{Y}$ & & \\
\hline Smittia foliacea $(\mathrm{K})$ & $F$ & & & & & & $\mathbf{Y}$ & Y & & Y & $\mathbf{Y}$ & & \\
\hline Smittia pratorum (G.) & & E & & & & & & & $\mathrm{E}$ & & Y & & \\
\hline Synorthocladius semivirens (K.) & & & & & & & & $\mathrm{Y}$ & & & & & \\
\hline Tvetenia verralli (Edw.) & $*$ & & & & & & & & & & $\mathbf{Y}$ & & \\
\hline \multicolumn{14}{|l|}{ Chironominae Chironomini } \\
\hline Chironomus phomosus (L.) & & & & & & & & & & & $\mathbf{Y}$ & & \\
\hline Chironomus pseudothummi Str. & & & & & & & & & & & & & $\mathrm{E}$ \\
\hline Chironomus riparius $\mathrm{Mg}$. & & & & & & & & & & & Y & Sk & \\
\hline Cladopelma edwardsi (Krus.) & * & & & & & & & & & & Y & & \\
\hline Cladopelma krusemani (G.) & * & & & & & & $Y$ & $Y$ & & Y & Y & & \\
\hline Endochironomus tendens (Fabr.) & * & & & & & Y & & & & & & & \\
\hline Microtendipes chloris (Mg.) & $*$ & & & & & & & & & & & Sk & \\
\hline Microtendipes pedellus (de Geer) & * & & & & & & & & & & Y & & \\
\hline Parachironomus parilis (Walk.) & $*$ & & & & & & & $\mathbf{Y}$ & & & & Sk & \\
\hline Paratendipes alhimanus (Mg.) & - & & & & & & $\mathrm{Y}$ & & & Y & & & \\
\hline Phaenopsectra flavipes (Mg.) & & & & & & $\mathbf{Y}$ & Y & & $\mathrm{Y}$ & Y & & & \\
\hline Potypedilum (Pentapedilum) nubens (Edw.) & $*$ & & & & & $\mathbf{Y}$ & & & & & & & \\
\hline Polypedilum (Pentapedilum) uncinatum (G.) & & & & & & & & & & & $\mathbf{Y}$ & & $\mathrm{E}$ \\
\hline Polypectilum (Uresipedilum) convicum (Walk.) & $\#$ & & & & & & & & & & Y & & \\
\hline Pohypedilum (s. str.) mubeculosum (Mg.) & & & & & & & & & & & & & $\mathrm{E}$ \\
\hline \multicolumn{14}{|l|}{ Chironominae Tanytarsini } \\
\hline Cladotanytarsus nigrovittatus (G.) & • & & & & & Y & & & & & & & \\
\hline Micropsectra atrofasciata $(\mathrm{K})$. & & & & & & $\mathrm{Y}$ & Y & & & Y & $\mathrm{Y}$ & & \\
\hline Micropsectra bidentata G. & * & & & & & & & & & & Y & " & \\
\hline Micropsectra fusca (Mg.) & & & & & & Y & $\mathrm{Y}$ & & $\mathrm{Y}$ & Y & Y. & & $\mathrm{E}$ \\
\hline Rheotwontarsus curtistylus (G.) & $*$ & & & 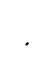 & & & & & & & Y & & \\
\hline Rheotanytarsus reissi Lehm. & $*$ & & & & & & & & & & $\mathrm{Y}$ & & \\
\hline Tamyiarsus pallidicomis (Walk) & & & & & & $\mathbf{Y}$ & & & & & & Sk & $\mathrm{E}$ \\
\hline Tanylarsus sylvaticus (van der Wulp) & - & & & & & $\mathrm{Y}$ & $\mathrm{Y}$ & & & Y & & & \\
\hline Zavrelia pentatoma $\mathrm{K}$ & $*$ & & & & & & & & & & $\mathbf{Y}$ & & \\
\hline
\end{tabular}




\section{References}

Burel F., Baudry J., Butet A., Clergeau P., Delettre Y.R., Le Cœur D., Dubs N., Morvan N., Paillat G., Petit S., Thenail C., Brunel E. \& Lefeuvre J.C. 1998. - Comparative biodiversity along a gradient of agricultural landscapes. Acta Oecol., $19: 47-60$.

Burel F., Baudry J., Delettre Y., Morvan N. \& Petit S. 2000. - Relating insects movements to farming systems in dynamic landscapes. In : Ekbom B., Irwin M. \& Robert M. Interchanges of insects between agricultural and surrounding landscapes. Kluwer Academic Publishers, The Netherlands : 5-32.

Cranston P.S. \& Oliver D.R. 1988. - Additions and corrections to the nearctic Orthocladiinae (Diptera : Chironomidae). Can. Entomol., $120: 425-462$.

Cranston P.S., Oliver D.R. \& Saether O.A. 1989. - The adult males of Orthocladiinae (Diptera : Chironomidae) of the Holarctic region. Keys and diagnoses. In : Wiederholm T. Chironomidae of the Holarctic region. Ent. scand., Suppl., 34 : 165-352.

Delettre Y.R. 1995. - Heathland fires temporarily increase species diversity : an example in terrestrial Chironomidae (Diptera). Landsc. Urban Plann., $31: 259-268$.

Delettre Y.R. \& Morvan N. 2000. - Dispersal of adult aquatic Chironomidae (Diptera) in agricultural landscapes. Freshwat. Biol., 44 : 399-411.

Delettre Y.R. 1984. — Recherches sur les Chironomides (Diptera) à larves édaphiques : biologie, écologie, mécanismes adaptatifs. Thèse Doctorat d'Etat. Université de Rennes I, $310 \mathrm{p}$.

Delettre Y.R. 1988. - Chironomid wing length, dispersal ability and habitat predictability. Holarct. Ecol., $11: 166-170$.

Delettre Y.R. 1988. - Flux d'évaporation corporelle et résistance à la dessiccation chez les larves de quelques Chironomidae terrestres (Diptera). Rev. Ecol. Biol. Sol, 25: 129-138.

Delettre Y.R. 1989. - Influence de la durée et de l'intensité de l'assèchement sur l'abondance et la phénologie des Chironomides (Diptera) d'une mare semi-permanente peu profonde. Archiv. $\mathrm{Hy}$ drobiol., $114:$ 383-399.

Delettre Y.R. 1993. - Terrestrial Chironomidae : contribution of local emergence to global aerial flow in heterogeneous environment. Neth. J. Aquat. Ecol., $26: 269-271$.

Delettre Y.R. 1994. - Fire disturbance of a chironomid (Diptera) community on heathlands. J. appl. Ecol., $31: 560-570$.

Delettre Y.R., Tréhen P. \& Grootaert P. 1992. - Space heterogeneity, space use and short-range dispersal in Diptera. A case study. Landsc. Ecol., $6: 175-181$.

Ducrotoy J.P. 1976. - Stratégies démographiques des populations de Chironomides (Diptera) d'une mare semi-permanente du massif forestier de Paimpont (Ille-et-Vilaine). Thèse de Doctorat de $3^{\mathrm{e}}$ cycle, Université de Rennes I : 144 p.

Fittkau E.J. \& Reiss F. 1978. - Chironomidae. In Illies J., Limnofauna europaea, Gustav. Fisher Verlag, Stuttgart, New York : 404-440.

Freeman P. 1955. - Diptera (Nematocera) : Chironomidae. In Hanström B., Brinck P. and Rudebeck G, South African animal life. II, Almquist \& Wiksell, Stockholm : 361-381.

Garcia X.-F. \& Laville H. 2001. - Importance of floodplain waters for the conservation of chironomid (Diptera) biodiversity in a $6^{\text {th }}$ order section of the Garonne river (France). Ann: Limnol., 37 : 3547.

Goddeeris L. \& Behen F. 1991. - Check-list of Belgian Chironomidae. In Grootaert P., De Bruyn L. \& De Meyer M.. Studiedocumenten van het K.B.I.N., 70 : 46-56.

Healey I.N. \& Russell-Smith A. 1971. - Abundance and feeding preferences of fly larvae in two woodland soils. Annales de Zoologie et d'Ecologie Animale 3, numéro hors-série, IV. Proc. 5th Colloquium pedobiologiae, Dijon, France : 177-192.

Illies J. 1978. - Limnofauna europaea. G. Fischer Verlag, $2^{\text {nd }}$. ed. : $532 \mathrm{p}$.
Langton P. 1991. - A key to pupal exuviae of West Palearctic Chironomidae. Privately published, Coleraine, N. Ireland : $386 \mathrm{p}$.

Laville H. \& Serra-Tosio B. 1996. - Additions et corrections à l'inventaire des Chironomidés (Diptera) de France depuis 1990. Annls. Limnol., 32 : 115-121.

Marmonier P., Claret C. \& Dole-Olivier M.J. 2000. - Interstitial communities of artificial drainage canals. Verh. Internat. Verein. Limnol., 27 : 438-441.

Moubayed J., Langton P.H. \& Morello E. 1999. - On some chironomid populations from permanent and temporary springs, streams and pools in Southern France: distribution and biogeographical significance. In Hoffrichter O. Late 20th century Research on Chironomidae : an Anthology from the 13th Int. Symp.Chir., Freiburg, Germany : 571-577.

Pinder L.C.V. 1978. - A key to adult males of British Chironomidae (Diptera). Sci. Publ. Freshwat. Biol. Ass., $37: 1-169$.

Rossaro B. 1988. - Revisione del genere Smittia Holmgren (Diptera, Chironomidae). la nota. Atti XV Congr. naz. ital. Ent. L'Aquila, Italia : 303-310.

Rossaro B. 1997. - Revision of the genus Smittia Holmgren, 1869, second note. Abstr. 13th International Symposium on Chironomidae Freiburg, Germany : 104.

Rossaro B. \& Delettre Y.R. 1992. - Description of Smittia celtica, n. sp. (Diptera, Chironomidae). Ann. Soc. ent. Fr, $28: 365-370$.

Saether O.A. 1973. - Four species of Bryophaenocladius Thien., with notes on other Orthocladiinae (Diptera : Chironomidae) Can. Entomol., 105 : 51-60.

Saether O.A. 1975. - Twelve new species of Limnophyes Eaton, with keys to nearctic males of the genus (Diptera : Chironomidae). Can. Entomol., 107 : 1029-1056.

Saether O.A. 1983. - A review of Holarctic Gymnometriocnemus Goetghebuer, with the description of Raphidocladius subgen. $\mathrm{n}$. and Sublettiella gen. n. (Diptera : Chironomidae). Aquat. Insects, $5: 209-226$.

Saether O.A. 1985a. - A review of the genus Limnophyes Eaton from the holarctic and afrotropical regions (Diptera : Chironomidae, Orthocladiinae). Ent. scand., Suppl., $35: 1-135$.

Saether O.A. 1985b. - A review of the genus Rheocricotopus Thienemann \& Harnisch 1932, with the description of three new species. Spixiana, Suppl., $11: 59-108$.

Saether O.A. 1989. - Metriocnemus van der Wulp : a new species and a revision of species described by Meigen, Zetterstedt, Staeger, Holmgren, Lundström and Strenzke (Diptera : Chironomidae): Ent. scand., $19: 393-430$.

Saether O. A. 1995. - Metriocnemus van der Wulp : Seven new species, revision of species, and new records (Diptera: Chironomidae). Annls. Limnol., $31: 35-64$.

Saether O.A., Godtfred A. \& Halvorsen A. 1981. - Diagnoses of Tvetenia Kieff., Dratnalia n. gen. and Eukiefferiella Thien. emend., with a phylogeny of the Cardiocladius group (Diptera : Chironomidae). Ent. scand., Suppl., $15: 269-285$.

Saether O.A. \& Wang X.H. 1995. - Revision of the genus Paraphaenocladius Thienemann, 1924, of the world (Diptera : Chironomidae, Orthocladiinae). Ent. scand., $48: 3-69$.

Serra-Tosio B.\& Laville H. 1991. - Liste annotée des Diptères Chironomidés de France continentale et de Corse. Annls. Limnol., $27: 37-74$.

Strenzke K. 1940. - Terrestrischen Chironomiden. V. Camptocladius stercorarius De Geer. Zool. Anz., 132 : 115-123.

Strenzke K. 1942. - Terrestrischen Chironomiden. XII. Bryophaenocladius subvernalis Edw. Zool. Anz., $137: 10-18$.

Thienemann K. \& Strenzke K. 1941. - Terrestrische Chironomiden. VII. Die Gattung Paraphaenocladius Thien. Zool. Anz., $133: 137-146$

Wilson R.S. \& McGill J.D. 1982. - A practical key to the genera of pupal exuviae of the British Chironomidae (Diptera, Insecta). University of Bristol, Library No. 0-86292-060-4: 62 p. 